\title{
BMC Structural Biology reviewer acknowledgment 2014
}

\section{Catherine J Potenski}

\author{
Oluwatoyin Asojo \\ USA
}

Shannon Au
Hong Kong
Lin Bai
USA
Douglas Barrick
USA

Nir Ben-Tal

Israel

Andrea Bernini

Italy

David Bevan

USA

Martino Bolognesi
Italy

Mohammad Reza Bozorgmehr

Iran

David Burke

UK

Teresa Carlomagno

Germany

Rita Casadio

Italy

Sudha Chakrapani

USA

Chi-Cheng Chiu

Taiwan

Patricia Coltri

Brazil

\section{Lisa Craig}

Canada

Nora Cronin
UK

Joel Eissenberg

USA

Jianwen Fang

USA

Diego Ferreiro

Argentina

Slawomir Filipek

Poland

Federico Fogolari

Italy

Dmitrij Frishman

Germany

Qingshan (Bill) Fu

USA

Bruce Gelb

USA

Vince Grolmusz

Kuwait

Nam-Chul Ha

Korea, South

Ulrich Hansmann

USA

Bart Hazes

Canada

Ronald Hills

USA
Gunnar Jeschke

Switzerland

Rameshwar Kadam

USA

Brian Kelch

USA

Chu-Young Kim

Singapore

Seiichiro Kishishita

Japan

Stephan König

Germany

Andriy Kryshtafovych

USA

Roman Laskowski

UK

Vanessa Leone

USA

Elena Levin

USA

Zhijun Li

USA

Christian Linke-Winnebeck

Germany

George Lountos

USA

Jianzhu Ma

USA

Francisco Martinez

Spain

Correspondence: catherine.potenski@biomedcentral.com

BioMed Central, 233 Spring Street, New York, NY 10013-1578, USA 
Yves Mechulam

France

Daniela Monti

Italy

Giulia Morra

Italy

Marvin Nieman

USA

Poul Nissen

Denmark

Diederik Opperman

South Africa

Yingjie Peng

USA
Robert Rambo

USA

Daniel Roche

France

Seong Eon Ryu

Korea, South

Wolf-Dieter Schubert

South Africa

Blake Simmons

USA

Heinz-Juergen Steinhoff

Germany

Eric Sundberg

USA
Dazhi Tan

USA

Silvio Tosatto

Italy

Tai Wang

Switzerland

Sheng Ye

China

Yaroslava Yingling

USA 